\title{
Estimation of $f$-divergence and Shannon entropy by Levinson type inequalities via new Green's functions and Lidstone polynomial
}

\author{
Muhammad Adeel ${ }^{1,2^{*}}$ (D) , Khuram Ali Khan ${ }^{1}$, Đilda Pečarić ${ }^{3}$ and Josip Pečarić ${ }^{4}$
}

\author{
"Correspondence: \\ adeel.uosmaths@gmail.com \\ ${ }^{1}$ Department of Mathematics, \\ University of Sargodha, Sargodha, \\ Pakistan \\ ${ }^{2}$ Department of Mathematics, \\ University of Central Punjab, Lahore, \\ Pakistan \\ Full list of author information is \\ available at the end of the article
}

\begin{abstract}
By using new Green's functions and Lidstone interpolating polynomial, some new generalizations of Levinson type inequalities for $(2 p+1)$-convex functions are obtained. In seek of applications of our results to information theory, new generalizations based on $f$-divergence estimates are also proven. Moreover, some inequalities for Shannon entropies are deduced as well.
\end{abstract}

Keywords: Information theory; Levinson's inequality; Lidstone interpolating polynomial; Green's functions

\section{Introduction and preliminaries}

The idea of Shannon entropy is the central job of information speculation, now and again implied as a measure of uncertainty. The entropy of a random variable is described with respect to a probability distribution, and it can be shown that it is a decent measure of random. The assignment of Shannon entropy is to assess the typical least number of bits expected to encode a progression of pictures subject to the letters, including the size and the repetition of the symbols.

Divergences between probability distributions can be interpreted as measures of distance between them. An assortment of sorts of divergences exist, for example the $\mathfrak{f}$ divergences (especially, Kullback-Leibler divergences, Hellinger distance, and total variation distance), Rényi divergences, Jensen-Shannon divergences, etc. (see $[1,2])$. There are a lot of papers dealing with the subject of inequalities and entropies, see, e.g., [3-7] and the references therein. Jensen's inequality deals with one kind of data points, Levinson's inequality deals with two types of data points.

\subsection{Csiszár divergence}

In $[8,9]$ Csiszár gave the following definition:

(c) The Author(s) 2020. This article is licensed under a Creative Commons Attribution 4.0 International License, which permits use sharing, adaptation, distribution and reproduction in any medium or format, as long as you give appropriate credit to the original author(s) and the source, provide a link to the Creative Commons licence, and indicate if changes were made. The images or other third party material in this article are included in the article's Creative Commons licence, unless indicated otherwise in a credit line to the material. If material is not included in the article's Creative Commons licence and your intended use is not permitted by statutory regulation or exceeds the permitted use, you will need to obtain permission directly from the copyright holder. To view a copy of this licence, visit http://creativecommons.org/licenses/by/4.0/. 
Definition 1 Let $f$ be a convex function from $\mathbb{R}^{+}$to $\mathbb{R}^{+}$. Let $\tilde{\mathbf{r}}, \tilde{\mathbf{k}} \in \mathbb{R}_{+}^{n}$ be such that $\sum_{\rho=1}^{n} r_{\rho}=1$ and $\sum_{\rho=1}^{n} k_{\rho}=1$. Then $f$-divergence functional is defined by

$$
\mathbb{I}_{f}(\tilde{\mathbf{r}}, \tilde{\mathbf{k}}):=\sum_{\rho=1}^{n} k_{\rho} f\left(\frac{r_{\rho}}{k_{\rho}}\right)
$$

By defining the following:

$$
f(0):=\lim _{x \rightarrow 0^{+}} f(x) ; \quad 0 f\left(\frac{0}{0}\right):=0 ; \quad 0 f\left(\frac{a}{0}\right):=\lim _{x \rightarrow 0^{+}} x f\left(\frac{a}{0}\right), \quad a>0,
$$

he stated that nonnegative probability distributions can also be used.

Using the definition of $f$-divergence functional, Horvath et al. [10] gave the following functional:

Definition 2 Let $\mathbb{I}$ be an interval contained in $\mathbb{R}$ and $f: \mathbb{I} \rightarrow \mathbb{R}$ be a function. Also let $\tilde{\mathbf{r}}=\left(r_{1}, \ldots, r_{n}\right) \in \mathbb{R}^{n}$ and $\tilde{\mathbf{k}}=\left(k_{1}, \ldots, k_{n}\right) \in(0, \infty)^{n}$ be such that

$$
\frac{r_{\rho}}{k_{\rho}} \in \mathbb{I}, \quad \rho=1, \ldots, n
$$

Then

$$
\hat{\mathbb{I}}_{f}(\tilde{\mathbf{r}}, \tilde{\mathbf{k}}):=\sum_{\rho=1}^{n} k_{\rho} f\left(\frac{r_{\rho}}{k_{\rho}}\right) .
$$

The theory of convex functions has encountered a fast advancement. This can be attributed to a few causes: firstly, applications of convex functions are directly involved in modern analysis; secondly, many important inequalities are results of applications of convex functions, and convex functions are closely related to inequalities (see [11]).

Divided differences are found to be very helpful when we are dealing with functions having different degrees of smoothness. The following definition of divided difference is given in [11, p. 14].

Levinson generalized Ky Fan's inequality for 3-convex in [12] (see also [13, p. 32, Theorem 1]) as follows:

Theorem 1 Let $f: \mathbb{I}=(0,2 \lambda) \rightarrow \mathbb{R}$ be such that $f$ is 3-convex. Also let $0<x_{\rho}<\lambda$ and $p_{\rho}>0$, then

$$
\begin{aligned}
\frac{1}{P_{n}} \sum_{\rho=1}^{n} p_{\rho} f\left(x_{\rho}\right)-f\left(\frac{1}{P_{n}} \sum_{\rho=1}^{n} p_{\rho} x_{\rho}\right) \leq & \frac{1}{P_{n}} \sum_{\rho=1}^{n} p_{\rho} f\left(2 \lambda-x_{\rho}\right) \\
& -f\left(\frac{1}{P_{n}} \sum_{\rho=1}^{n} p_{\rho}\left(2 \lambda-x_{\rho}\right)\right) .
\end{aligned}
$$


Inequality (2) gives us the following functional:

$$
\begin{aligned}
J_{1}(f(\cdot))= & \frac{1}{P_{n}} \sum_{\rho=1}^{n} p_{\rho} f\left(2 \lambda-x_{\rho}\right)-f\left(\frac{1}{P_{n}} \sum_{\rho=1}^{n} p_{\rho}\left(2 \lambda-x_{\rho}\right)\right)-\frac{1}{P_{n}} \sum_{\rho=1}^{n} p_{\rho} f\left(x_{\rho}\right) \\
& +f\left(\frac{1}{P_{n}} \sum_{\rho=1}^{n} p_{\rho} x_{\rho}\right) \geq 0 .
\end{aligned}
$$

In [14], Popoviciu noticed that Levinson's inequality (2) is substantial on $(0,2 \lambda)$ for 3convex functions, while in [15] (see additionally [13, p. 32, Theorem 2]) Bullen gave distinctive confirmation of Popoviciu's result and furthermore the converse of (2).

\section{Theorem 2}

(a) Let $f: \mathbb{I}=\left[\zeta_{1}, \zeta_{2}\right] \rightarrow \mathbb{R}$ be a 3-convex function and $x_{\rho}, y_{\rho} \in\left[\zeta_{1}, \zeta_{2}\right]$ for $\rho=1,2, \ldots, n$ be such that

$$
\max \left\{x_{1} \cdots x_{n}\right\} \leq \min \left\{y_{1} \cdots y_{n}\right\}, \quad x_{1}+y_{1}=\cdots=x_{n}+y_{n}
$$

and $p_{\rho}>0$, then

$$
\frac{1}{P_{n}} \sum_{\rho=1}^{n} p_{\rho} f\left(x_{\rho}\right)-f\left(\frac{1}{P_{n}} \sum_{\rho=1}^{n} p_{\rho} x_{\rho}\right) \leq \frac{1}{P_{n}} \sum_{\rho=1}^{n} p_{\rho} f\left(y_{\rho}\right)-f\left(\frac{1}{P_{n}} \sum_{\rho=1}^{n} p_{\rho} y_{\rho}\right)
$$

(b) If $p_{\rho}>0$, inequality (5) is valid for all $x_{\rho}, y_{\rho}$ satisfying condition (4), and function $f$ is continuous, then $f$ is 3-convex.

The following functional arises from inequality (5):

$$
\begin{aligned}
J_{2}(f(\cdot))= & \frac{1}{P_{n}} \sum_{\rho=1}^{n} p_{\rho} f\left(y_{\rho}\right)-f\left(\frac{1}{P_{n}} \sum_{\rho=1}^{n} p_{\rho} y_{\rho}\right)-\frac{1}{P_{n}} \sum_{\rho=1}^{n} p_{\rho} f\left(x_{\rho}\right) \\
& +f\left(\frac{1}{P_{n}} \sum_{\rho=1}^{n} p_{\rho} x_{\rho}\right) \geq 0 .
\end{aligned}
$$

Remark 1 In the above results, if the function $f$ is 3 -convex, then $J_{k}(f(\cdot)) \geq 0$ for $k=1,2$ and $J_{k}(f(\cdot))=0$ for $f(x)=x$ or $f(x)=x^{2}$ or a constant function $f$.

In the following result, Pečarić [16] (see also [13, p. 32, Theorem 4]) proved inequality (5) by weakening condition (4).

Theorem 3 Let $f: \mathbb{I}=\left[\zeta_{1}, \zeta_{2}\right] \rightarrow \mathbb{R}$ be such that $f^{3}(t) \geq 0, p_{\rho}>0$. Also let $x_{\rho}, y_{\rho} \in\left[\zeta_{1}, \zeta_{2}\right]$ be such that $x_{\rho}+y_{\rho}=2 \breve{c}$ for $\rho=1, \ldots, n, x_{\rho}+x_{n-\rho+1} \leq 2 \breve{c}$ and $\frac{p_{\rho} x_{\rho}+p_{n-\rho+1} x_{n-\rho+1}}{p_{\rho}+p_{n-\rho+1}} \leq \breve{c}$. Then inequality (5) holds.

In [17], Mercer proved that inequality (5) still holds after replacing the symmetry condition with symmetric variances of points. His result is given in the following theorem. 
Theorem 4 Let $f: \mathbb{I}=\left[\zeta_{1}, \zeta_{2}\right] \rightarrow \mathbb{R}$ be such that $f^{3}(t) \geq 0$, $p_{\rho}$ are positive such that $\sum_{\rho=1}^{n} p_{\rho}=1$. Also, let $x_{\rho}, y_{\rho}$ satisfy $\max \left\{x_{1} \cdots x_{\rho}\right\} \leq \min \left\{y_{1} \cdots y_{\rho}\right\}$ and

$$
\sum_{\rho=1}^{n} p_{\rho}\left(x_{\rho}-\sum_{\rho=1}^{n} p_{\rho} x_{\rho}\right)^{2}=\sum_{\rho=1}^{n} p_{\rho}\left(y_{\rho}-\sum_{\rho=1}^{n} p_{\rho} y_{\rho}\right)^{2}
$$

then (5) holds.

Lidstone polynomials are useful in literature to generalize a number of novel inequalities including Jensen, Ostrowski, Chebysev, and Hermite-Hadamard type inequalities. In the literature, several extensions and generalizations of the said inequalities are found via Lidstone interpolation. However, all these results involve only one type of data points and are for the class of convex functions along with generalization for $(2 p)$-convex functions.

The following result was proved by Wider in [18]:

Lemma 1.1 If $\in \in C^{\infty}[0,1]$, then

$$
f(t)=\sum_{l=0}^{p-1}\left[f^{(2 l)}(0) \Theta_{l}(1-t)+f^{(2 l)}(0) \Theta_{l}(t)\right]+\int_{0}^{1} G_{p}(t, s) f^{(2 p)}(t) d t
$$

where $\Theta_{l}$ is a polynomial of degree $(2 l+1)$ defined by the relations

$$
\Theta_{0}(t)=t, \quad \Theta_{p}^{\prime \prime}(t)=\Theta_{p-1}(t), \quad \Theta_{p}(0)=\Theta_{p}(1)=0, \quad p \geq 1,
$$

and

$$
G_{1}(t, s)=G(t, s)= \begin{cases}(t-1) s, & s \leq t \\ (s-1) t, & t \leq s\end{cases}
$$

is homogeneous Green's function of the differential operator $\frac{d^{2}}{d s^{2}}$ on $[0,1]$, and with the successive iterates of $G(t, s)$

$$
G_{p}(t, s)=\int_{0}^{1} G_{1}(t, k) G_{p-1}(k, s) d k, \quad p \geq 2 .
$$

The Lidstone polynomial can be expressed in terms of $G_{p}(t, s)$ as

$$
\Theta_{p}(t)=\int_{0}^{1} G_{p}(t, s) s d s
$$

The Lidstone series representation of $f \in C^{2 p}\left[\zeta_{1}, \zeta_{2}\right]$ is given in [19] as follows:

$$
\begin{aligned}
f(x)= & \sum_{l=0}^{p-1}\left(\zeta_{2}-\zeta_{1}\right)^{2 l} f^{(2 l)}\left(\zeta_{1}\right) \Theta_{l}\left(\frac{\zeta_{2}-x}{\zeta_{2}-\zeta_{1}}\right)+\sum_{l=0}^{p-1}\left(\zeta_{2}-\zeta_{1}\right)^{2 l} f^{(2 l)}\left(\zeta_{2}\right) \Theta_{l}\left(\frac{x-\zeta_{1}}{\zeta_{2}-\zeta_{1}}\right) \\
& +\left(\zeta_{2}-\zeta_{1}\right)^{2 p-1} \int_{\zeta_{1}}^{\zeta_{2}} G_{p}\left(\frac{x-\zeta_{1}}{\zeta_{2}-\zeta_{1}}, \frac{t-\zeta_{1}}{\zeta_{2}-\zeta_{1}}\right) f^{(2 p)}(t) d t
\end{aligned}
$$


The error function $e_{\mathcal{F}}(t)$ can be represented in terms of Green's function $G_{\mathcal{F}, n}(t, s)$ of the boundary value problem

$$
\begin{aligned}
& z^{(n)}(t)=0, \\
& z^{(i)}\left(\zeta_{1}\right)=0, \quad 0 \leq i \leq p, \\
& z^{(i)}\left(\zeta_{2}\right)=0, \quad p+1 \leq i \leq n-1, \\
& e_{F}(t)=\int_{\zeta_{1}}^{\zeta_{2}} G_{F, n}(t, s) f^{(n)}(s) d s, \quad t \in\left[\zeta_{1}, \zeta_{2}\right],
\end{aligned}
$$

where

$$
G_{F, n}(t, s)=\frac{1}{(n-1) !} \begin{cases}\sum_{i=0}^{p}\left(\begin{array}{c}
n-1 \\
i
\end{array}\right)\left(t-\zeta_{1}\right)^{i}\left(\zeta_{1}-s\right)^{n-i-1}, & \zeta_{1} \leq s \leq t \\
-\sum_{i=p+1}^{n-1}\left(\begin{array}{c}
n-1 \\
i
\end{array}\right)\left(t-\zeta_{1}\right)^{i}\left(\zeta_{1}-s\right)^{n-i-1}, & t \leq s \leq \zeta_{2}\end{cases}
$$

In [20] Aras Gazić et al. proved the following result:

Theorem 5 Let $f \in C^{n}\left[\zeta_{1}, \zeta_{2}\right]$ and $P_{F}$ be its 'two-point right focal' interpolating polynomial. Then, for $\zeta_{1} \leq a_{1}<a_{2} \leq \zeta_{2}$ and $0 \leq p \leq n-2$,

$$
\begin{aligned}
f(t)= & P_{F}(t)+e_{F}(t) \\
= & \sum_{i=0}^{p} \frac{\left(t-a_{1}\right)^{i}}{i !} f^{(i)}\left(a_{1}\right) \\
& +\sum_{j=0}^{n-p-2}\left(\sum_{i=0}^{j} \frac{\left(t-a_{1}\right)^{p+1+i}\left(a_{1}-a_{2}\right)^{j-i}}{(p+1+i) !(j-i) !}\right) f^{(p+1+j)}\left(a_{2}\right) \\
& +\int_{a_{1}}^{a_{2}} G_{F, n}(t, s) f^{(n)}(s) d s,
\end{aligned}
$$

where $G_{F, n}(t, s)$ is the Green's function defined by (12).

We have the following two cases from (13).

(Case-1) For $n=3$ and $p=0$

$$
\begin{aligned}
f(t)= & f\left(a_{1}\right)+\left(t-a_{1}\right) f^{(1)}\left(a_{2}\right)+\left(t-a_{1}\right)\left(a_{1}-a_{2}\right) f^{(2)}\left(a_{2}\right)+\frac{\left(t-a_{1}\right)^{2}}{2} f^{(2)}\left(a_{2}\right) \\
& +\int_{a_{1}}^{a_{2}} G_{1}(t, s) f^{(3)}(s) d s
\end{aligned}
$$

where

$$
G_{1}(t, s)= \begin{cases}\left(a_{1}-s\right)^{2}, & a_{1} \leq s \leq t \\ -\left(t-a_{1}\right)\left(a_{1}-s\right)+\frac{1}{2}\left(t-a_{1}\right)^{2}, & t \leq s \leq a_{2}\end{cases}
$$

(Case-2) For $n=3$ and $p=1$

$$
f(t)=f\left(a_{1}\right)+\left(t-a_{1}\right) f^{(1)}\left(a_{2}\right)+\frac{\left(t-a_{1}\right)^{2}}{2} f^{(2)}\left(a_{2}\right)+\int_{a_{1}}^{a_{2}} G_{2}(t, s) f^{(3)}(s) d s,
$$


where

$$
G_{2}(t, s)= \begin{cases}\frac{1}{2}\left(a_{1}-s\right)^{2}+\left(t-a_{1}\right)\left(a_{1}-s\right), & a_{1} \leq s \leq t \\ -\frac{1}{2}\left(t-a_{1}\right)^{2}, & t \leq s \leq a_{2}\end{cases}
$$

In [21], Pečarić et al. gave a probabilistic version of inequality (2) under condition (7). In [22] an operator version of probabilistic Levinson's inequality was discussed. In [20], Gazić et al. considered the class of $2 p$-convex functions and generalized Jensen's inequality and converses of Jensen's inequality by using Lidstone's interpolating polynomials. All generalizations that exist in literature refer only to one type of data points. But in this paper, motivated by the above discussion, Levinson type inequalities are generalized for $(2 p+1)$ convex function via Lidstone interpolating polynomial involving two types of data points for higher order convex functions.

\section{Main results}

Motivated by functional (6), we generalize the following new results with the help of Lidstone interpolating polynomial given by (11).

\subsection{Generalization of Bullen type inequalities for $(2 p+1)$-convex functions}

First we define the following functional:

$\mathcal{F}:$ Let $f: \mathbb{I}_{1}=\left[\zeta_{1}, \zeta_{2}\right] \rightarrow \mathbb{R}$ be a function, $x_{1}, \ldots, x_{n}$ and $y_{1}, \ldots, y_{m} \in \mathbb{I}_{1}$ be such that

$$
\max \left\{x_{1} \cdots x_{n}\right\} \leq \min \left\{y_{1} \cdots y_{m}\right\}, \quad x_{1}+y_{1}=\cdots=x_{n}+y_{m} .
$$

Also let $\left(p_{1}, \ldots, p_{n}\right) \in \mathbb{R}^{n}$ and $\left(q_{1}, \ldots, q_{m}\right) \in \mathbb{R}^{m}$ be such that $\sum_{\rho=1}^{n} p_{\rho}=1 \sum_{\varrho=1}^{m} q_{\varrho}=1$ and $x_{\rho}, y_{\varrho}, \sum_{\rho=1}^{n} p_{\rho} x_{\rho}, \sum_{\varrho=1}^{m} q_{\varrho} y_{\varrho} \in \mathbb{I}_{1}$. Then

$$
\breve{J}(f(\cdot))=\sum_{\varrho=1}^{m} q_{\varrho} f\left(y_{\varrho}\right)-f\left(\sum_{\varrho=1}^{m} q_{\varrho} y_{\varrho}\right)-\sum_{\rho=1}^{n} p_{\rho} f\left(x_{\rho}\right)+f\left(\sum_{\rho=1}^{n} p_{\rho} x_{\rho}\right) .
$$

Theorem 6 Assume $\mathcal{F}$ with $f \in C^{2 p+1}\left[\zeta_{1}, \zeta_{2}\right]$, and let $\Theta_{p}(t)$ be the same as defined in Lemma 1.1. Then

$$
\begin{aligned}
\breve{J}(f(\cdot))= & \sum_{l=0}^{p-2}\left(\zeta_{2}-\zeta_{1}\right)^{2 l}\left[f^{(2 l+3)}\left(\zeta_{1}\right) \int_{\zeta_{1}}^{\zeta_{2}} \breve{J}\left(G_{k}(\cdot, s)\right) \Theta_{l}\left(\frac{\zeta_{2}-s}{\zeta_{2}-\zeta_{1}}\right) d s\right. \\
& \left.+f^{(2 l+3)}\left(\zeta_{2}\right) \int_{\zeta_{1}}^{\zeta_{2}} \breve{J}\left(G_{k}(\cdot, s)\right) \Theta_{l}\left(\frac{s-\zeta_{1}}{\zeta_{2}-\zeta_{1}}\right) d s\right] \\
& +\left(\zeta_{2}-\zeta_{1}\right)^{2 p-3} \int_{\zeta_{1}}^{\zeta_{2}} f^{(2 p+1)}(v) \\
& \times\left(\int_{\zeta_{1}}^{\zeta_{2}} \breve{J}\left(G_{k}(\cdot, s)\right) G_{p}\left(\frac{s-\zeta_{1}}{\zeta_{2}-\zeta_{1}}, \frac{v-\zeta_{1}}{\zeta_{2}-\zeta_{1}}\right) d s\right) d v,
\end{aligned}
$$


where

$$
\begin{aligned}
\breve{J}\left(G_{k}(\cdot, s)\right)= & \sum_{\varrho=1}^{m} q_{\varrho} G_{k}\left(y_{\varrho}, s\right)-G_{k}\left(\sum_{\varrho=1}^{m} q_{\varrho}\left(y_{\varrho}, s\right)\right) \\
& -\sum_{\rho=1}^{n} p_{\rho} G_{k}\left(x_{\rho}, s\right)+G_{k}\left(\sum_{\rho=1}^{n} p_{\rho} x_{\rho}, s\right)
\end{aligned}
$$

and $G_{k}(\cdot, s)(k=1,2)$ are defined in (15) and (17) respectively.

Proof Applying (19) to identities (14) and (16) respectively along with there defined new Green's functions, by means of simple calculations and following the properties of $\breve{J}(f(\cdot))$, we get

$$
\breve{J}(f(\cdot))=\int_{\zeta_{1}}^{\zeta_{2}} \breve{J}\left(G_{k}(\cdot, s)\right) f^{(3)}(s) d s .
$$

Using Lidstone series representation (11) on the function $f^{(3)}(s)$, we have

$$
\begin{aligned}
f^{(3)}(s)= & \sum_{l=0}^{p-1}\left(\zeta_{2}-\zeta_{1}\right)^{2 l} f^{(2 l+3)}\left(\zeta_{1}\right) \Theta_{l}\left(\frac{\zeta_{2}-s}{\zeta_{2}-\zeta_{1}}\right)+\sum_{l=0}^{p-1}\left(\zeta_{2}-\zeta_{1}\right)^{2 l} f^{(2 l+3)}\left(\zeta_{2}\right) \\
& \times \Theta_{l}\left(\frac{s-\zeta_{1}}{\zeta_{2}-\zeta_{1}}\right)+\left(\zeta_{2}-\zeta_{1}\right)^{2 p-1} \int_{\zeta_{1}}^{\zeta_{2}} G_{p}\left(\frac{s-\zeta_{1}}{\zeta_{2}-\zeta_{1}}, \frac{v-\zeta_{1}}{\zeta_{2}-\zeta_{1}}\right) f^{(2 p+3)}(v) d v
\end{aligned}
$$

Replacing $p$ by $p-1$, we get

$$
\begin{aligned}
f^{(3)}(s)= & \sum_{l=0}^{p-2}\left(\zeta_{2}-\zeta_{1}\right)^{2 l}\left(f^{(2 l+3)}\left(\zeta_{1}\right) \Theta_{l}\left(\frac{\zeta_{2}-s}{\zeta_{2}-\zeta_{1}}\right)+f^{(2 l+3)}\left(\zeta_{2}\right) \Theta_{l}\left(\frac{s-\zeta_{1}}{\zeta_{2}-\zeta_{1}}\right)\right) \\
& +\left(\zeta_{2}-\zeta_{1}\right)^{2 p-3} \int_{\zeta_{1}}^{\zeta_{2}} G_{p}\left(\frac{s-\zeta_{1}}{\zeta_{2}-\zeta_{1}}, \frac{\nu-\zeta_{1}}{\zeta_{2}-\zeta_{1}}\right) f^{(2 p+1)}(v) d v
\end{aligned}
$$

Now, using (23) in (22) yields

$$
\begin{aligned}
\breve{J}(f(\cdot))= & \int_{\zeta_{1}}^{\zeta_{2}} \breve{J}\left(G_{k}(\cdot, s)\right)\left[\sum _ { l = 0 } ^ { p - 2 } ( \zeta _ { 2 } - \zeta _ { 1 } ) ^ { 2 l } \left(f^{(2 l+3)}\left(\zeta_{1}\right) \Theta_{l}\left(\frac{\zeta_{2}-s}{\zeta_{2}-\zeta_{1}}\right)\right.\right. \\
& \left.+f^{(2 l+3)}\left(\zeta_{2}\right) \Theta_{l}\left(\frac{s-\zeta_{1}}{\zeta_{2}-\zeta_{1}}\right)\right)+\left(\zeta_{2}-\zeta_{1}\right)^{2 p-3} \\
& \left.\times \int_{\zeta_{1}}^{\zeta_{2}} G_{p}\left(\frac{s-\zeta_{1}}{\zeta_{2}-\zeta_{1}}, \frac{v-\zeta_{1}}{\zeta_{2}-\zeta_{1}}\right) f^{(2 p+1)}(v) d v\right] d s .
\end{aligned}
$$

After rearranging the terms in (24), we have

$$
\begin{aligned}
\breve{J}(f(\cdot))= & \sum_{l=0}^{p-2}\left(\zeta_{2}-\zeta_{1}\right)^{2 l}\left[f^{(2 l+3)}\left(\zeta_{1}\right) \int_{\zeta_{1}}^{\zeta_{2}} \breve{J}\left(G_{k}(\cdot, s)\right) \Theta_{l}\left(\frac{\zeta_{2}-s}{\zeta_{2}-\zeta_{1}}\right) d s\right. \\
& \left.+f^{(2 l+3)}\left(\zeta_{2}\right) \int_{\zeta_{1}}^{\zeta_{2}} \breve{J}\left(G_{k}(\cdot, s)\right) \Theta_{l}\left(\frac{s-\zeta_{1}}{\zeta_{2}-\zeta_{1}}\right) d s\right]
\end{aligned}
$$




$$
\begin{aligned}
& +\left(\zeta_{2}-\zeta_{1}\right)^{2 p-3} \int_{\zeta_{1}}^{\zeta_{2}} \breve{J}\left(G_{k}(\cdot, s)\right) \\
& \times\left(\int_{\zeta_{1}}^{\zeta_{2}} G_{p}\left(\frac{s-\zeta_{1}}{\zeta_{2}-\zeta_{1}}, \frac{v-\zeta_{1}}{\zeta_{2}-\zeta_{1}}\right) f^{(2 p+1)}(v) d v\right) d s .
\end{aligned}
$$

Executing Fubini's theorem in the last term of (25) yields (20).

As an application we obtain Bullen type inequality for $(2 p+1)$-convex functions.

Theorem 7 Assume that all the conditions of Theorem 6 hold, and let $f$ be $a(2 p+1)$-convex function. Then, for $k=1,2$, we have the following result:

If

$$
\int_{\zeta_{1}}^{\zeta_{2}} \breve{J}\left(G_{k}(\cdot, s)\right) G_{p}\left(\frac{s-\zeta_{1}}{\zeta_{2}-\zeta_{1}}, \frac{v-\zeta_{1}}{\zeta_{2}-\zeta_{1}}\right) d s \geq 0
$$

then

$$
\begin{aligned}
\breve{J}(f(\cdot)) \geq & \sum_{l=0}^{p-2}\left(\zeta_{2}-\zeta_{1}\right)^{2 l}\left[f^{(2 l+3)}\left(\zeta_{1}\right) \int_{\zeta_{1}}^{\zeta_{2}} \breve{J}\left(G_{k}(\cdot, s)\right) \Theta_{l}\left(\frac{\zeta_{2}-s}{\zeta_{2}-\zeta_{1}}\right) d s\right. \\
& \left.+f^{(2 l+3)}\left(\zeta_{2}\right) \int_{\zeta_{1}}^{\zeta_{2}} \breve{J}\left(G_{k}(\cdot, s)\right) \Theta_{l}\left(\frac{s-\zeta_{1}}{\zeta_{2}-\zeta_{1}}\right) d s\right] .
\end{aligned}
$$

Proof Since the function $f$ is $(2 p+1)$-convex and is $(2 p+1)$ times differentiable, we have

$$
f^{(2 p+1)}(x) \geq 0 \quad \forall x \in \mathbb{I}_{1}
$$

therefore, by using (26) in (27), we get the required results respectively.

\section{Remark 2}

(i) In Theorem 7, inequality (26) holds in reverse direction if the inequality in (27) is reversed.

(ii) Inequality in (27) is also reversed if $f$ is $(2 p+1)$-concave.

If we put $m=n, p_{\rho}=q_{\varrho}$ and use positive weights in (19), then $\breve{J}(\cdot)$ converts to the functional $J_{2}(\cdot)$ defined in (6), also in this case (20), (21), (26), and (27) become

$$
\begin{aligned}
J_{2}(f(\cdot))= & \sum_{l=0}^{p-2}\left(\zeta_{2}-\zeta_{1}\right)^{2 l}\left[f^{(2 l+3)}\left(\zeta_{1}\right) \int_{\zeta_{1}}^{\zeta_{2}} J_{2}\left(G_{k}(\cdot, s)\right) \Theta_{l}\left(\frac{\zeta_{2}-s}{\zeta_{2}-\zeta_{1}}\right) d s\right. \\
& \left.+f^{(2 l+3)}\left(\zeta_{2}\right) \int_{\zeta_{1}}^{\zeta_{2}} J_{2}\left(G_{k}(\cdot, s)\right) \Theta_{l}\left(\frac{s-\zeta_{1}}{\zeta_{2}-\zeta_{1}}\right) d s\right] \\
& +\left(\zeta_{2}-\zeta_{1}\right)^{2 p-3} \int_{\zeta_{1}}^{\zeta_{2}} f^{(2 p+1)}(v) \\
& \times\left(\int_{\zeta_{1}}^{\zeta_{2}} J_{2}\left(G_{k}(\cdot, s)\right) G_{p}\left(\frac{s-\zeta_{1}}{\zeta_{2}-\zeta_{1}}, \frac{v-\zeta_{1}}{\zeta_{2}-\zeta_{1}}\right) d s\right) d v
\end{aligned}
$$




$$
\begin{aligned}
& J_{2}\left(G_{k}(\cdot, s)\right)= \sum_{\rho=1}^{n} p_{\rho} G_{k}\left(y_{\rho}, s\right)-G_{k}\left(\sum_{\rho=1}^{n} p_{\rho}\left(y_{\rho}, s\right)\right) \\
&-\sum_{\rho=1}^{n} p_{\rho} G_{k}\left(x_{\rho}, s\right)+G_{k}\left(\sum_{\rho=1}^{n} p_{\rho} x_{\rho}, s\right), \\
& \int_{\zeta_{1}}^{\zeta_{2}} J_{2}\left(G_{k}(\cdot, s)\right) G_{p}\left(\frac{s-\zeta_{1}}{\zeta_{2}-\zeta_{1}}, \frac{v-\zeta_{1}}{\zeta_{2}-\zeta_{1}}\right) d s \geq 0,
\end{aligned}
$$

and

$$
\begin{aligned}
J_{2}(f(\cdot)) \geq & \sum_{l=0}^{p-2}\left(\zeta_{2}-\zeta_{1}\right)^{2 l}\left[f^{(2 l+3)}\left(\zeta_{1}\right) \int_{\zeta_{1}}^{\zeta_{2}} J_{2}\left(G_{k}(\cdot, s)\right) \Theta_{l}\left(\frac{\zeta_{2}-s}{\zeta_{2}-\zeta_{1}}\right) d s\right. \\
& \left.+f^{(2 l+3)}\left(\zeta_{2}\right) \int_{\zeta_{1}}^{\zeta_{2}} J_{2}\left(G_{k}(\cdot, s)\right) \Theta_{l}\left(\frac{s-\zeta_{1}}{\zeta_{2}-\zeta_{1}}\right) d s\right] .
\end{aligned}
$$

Theorem 8 Let $f: \mathbb{I}_{1}=\left[\zeta_{1}, \zeta_{2}\right] \rightarrow \mathbb{R}$ be a $(2 p+1)$-convex function. Also let $\left(p_{1}, \ldots, p_{n}\right)$ be positive real numbers such that $\sum_{\rho=1}^{n} p_{\rho}=1$. Then, for the functional $J_{2}(\cdot)$ defined in (6), and using $\Theta_{p}(t)$ defined in Lemma 1.1, we have the following:

(i) For $k=1,2$, inequality (31) holds provided that $p$ is odd.

(ii) For fixed $k=1,2$, let inequality (31) be satisfied and

$$
\sum_{l=0}^{p-2}\left(\zeta_{2}-\zeta_{1}\right)^{2 l}\left[f^{(2 l+3)}\left(\zeta_{1}\right) \Theta_{l}\left(\frac{\zeta_{2}-s}{\zeta_{2}-\zeta_{1}}\right)+f^{(2 l+3)}\left(\zeta_{2}\right) \Theta_{l}\left(\frac{s-\zeta_{1}}{\zeta_{2}-\zeta_{1}}\right)\right] \geq 0
$$

Then

$$
J_{2}(f(\cdot)) \geq 0
$$

Proof It is clear that Green's functions $G_{k}(\cdot, s)$ defined in (15) and (17) are 3-convex and the weights are assumed to be positive. Therefore, applying Theorem 2 and using Remark 1 , we have $J_{2}\left(G_{k}(\cdot, s)\right) \geq 0$ for fixed $k=1,2$.

(i) $G_{p}\left(\frac{s-\zeta_{1}}{\zeta_{2}-\zeta_{1}}, \frac{v-\zeta_{1}}{\zeta_{2}-\zeta_{1}}\right) \geq 0$ for odd $p$, therefore (30) holds. As $f$ is $(2 p+1)$-convex, hence, by following Theorem 7, we get (31).

(ii) Using (32) in (31), we get (33) for fixed $k=1,2$.

In the next result we give a generalization of the Levinson type inequality given in [16] (see also [13]).

Theorem 9 Let $f \in C^{2 p+1}\left[\zeta_{1}, \zeta_{2}\right],\left(p_{1}, \ldots, p_{n}\right) \in \mathbb{R}^{n},\left(q_{1}, \ldots, q_{m}\right) \in \mathbb{R}^{m}$ be such that $\sum_{\rho=1}^{n} p_{\rho}=$ $1, \sum_{\varrho=1}^{m} q_{\varrho}=1$ and $\sum_{\varrho=1}^{m} q_{\varrho} y_{\varrho}$ and $\sum_{\rho=1}^{n} p_{\rho} x_{\rho} \in \mathbb{I}_{1}$. Also let $x_{1}, \ldots, x_{n}$ and $y_{1}, \ldots, y_{m} \in \mathbb{I}_{1}$ be such that $x_{\rho}+y_{\varrho}=2 \breve{c}$ and $x_{\rho}+x_{n-\rho+1} \leq 2 \breve{c}, \frac{p_{\rho} x_{\rho}+p_{n-\rho+1} x_{n-\rho+1}}{p_{\rho}+p_{n-\rho+1}} \leq \breve{c}$. Moreover, let $\Theta_{p}(t)$ be the same as defined in Lemma 1.1, then (20) holds.

Proof Proof is similar to Theorem 6 by assuming conditions given in the statement.

As an application, we obtain generalizations of Levinson type functional for $(2 p+1)$ convex functions $(p>1)$. 
Theorem 10 Let $f \in C^{2 p+1}\left[\zeta_{1}, \zeta_{2}\right](p>1),\left(p_{1}, \ldots, p_{n}\right) \in \mathbb{R}^{n},\left(q_{1}, \ldots, q_{m}\right) \in \mathbb{R}^{m}$ be such that $\sum_{\rho=1}^{n} p_{\rho}=1, \sum_{\varrho=1}^{m} q_{\varrho}=1$ and $\sum_{\varrho=1}^{m} q_{\varrho} y_{\varrho}$ and $\sum_{\rho=1}^{n} p_{\rho} x_{\rho} \in \mathbb{I}_{1}$. Also let $x_{1}, \ldots, x_{n}$ and $y_{1}, \ldots, y_{m} \in \mathbb{I}_{1}$ be such that $x_{\rho}+y_{\varrho}=2 \breve{c}$ and $x_{\rho}+x_{n-\rho+1} \leq 2 \breve{c}, \frac{p_{\rho} x_{\rho}+p_{n-\rho+1} x_{n-\rho+1}}{p_{\rho}+p_{n-\rho+1}} \leq \breve{c}$. Moreover, let $\Theta_{p}(t)$ be the same as defined in Lemma 1.1. If (26) is valid, then (27) is also valid.

Proof Proof is similar to Theorem 7.

Theorem 11 Let $f \in C^{2 p+1}\left[\zeta_{1}, \zeta_{2}\right](p>1),\left(p_{1}, \ldots, p_{n}\right)$ be positive real numbers such that $\sum_{\rho=1}^{n} p_{\rho}=1$. Also let $x_{1}, \ldots, x_{n}$ and $y_{1}, \ldots, y_{n} \in \mathbb{I}_{1}$ be such that $x_{\rho}+y_{\varrho}=2 \breve{c}$ and $x_{\rho}+x_{n-\rho+1}$, $\frac{p_{\rho} x_{\rho}+p_{n-\rho+1} x_{n-\rho+1}}{p_{\rho}+p_{n-\rho+1}} \leq \breve{c}$. Moreover, let $\Theta_{p}(t)$ be the same as defined in Lemma 1.1. Then

(i) For $k=1,2$, inequality (31) holds provided that $p$ is odd.

(ii) For fixed $k=1,2$, let inequality (31) be satisfied, then (33) holds.

Proof Proof is similar to Theorem 10.

In the next result, a Levinson type inequality is given (for positive weights) under Mercer's condition (7).

Corollary 1 Let $f: \mathbb{I}_{1}=\left[\zeta_{1}, \zeta_{2}\right] \rightarrow \mathbb{R}$ be a $(2 p+1)$-convex function, $x_{\rho}, y_{\rho}$ satisfy $(7)$ and $\max \left\{x_{1} \cdots x_{n}\right\} \leq \min \left\{y_{1} \cdots y_{n}\right\}$. Also let $p_{\rho}$ be such that $\sum_{\rho=1}^{n} p_{\rho}=1$. Moreover, let $\Theta_{p}(t)$ be the same as defined in Lemma 1.1. Then (28) holds.

Proof We get (28) after using the conditions given in the statement and following similar steps as in the proof of Theorem 6.

\subsection{Generalization of Levinson type inequality for $(2 p+1)$-convex functions}

Motivated by functional (3), we generalize the following new results with the help of Lidstone interpolating polynomial given by (11).

First we defined the following functional:

$\mathcal{H}:$ Let $f: \mathbb{I}_{2}=[0,2 a] \rightarrow \mathbb{R}$ be a function, $x_{1}, \ldots, x_{n} \in(0, a),\left(p_{1}, \ldots, p_{n}\right) \in \mathbb{R}^{n},\left(q_{1}, \ldots, q_{m}\right) \in$ $\mathbb{R}^{m}$ are real numbers such that $\sum_{\rho=1}^{n} p_{\rho}=1$ and $\sum_{\varrho=1}^{m} q_{\varrho}=1$. Also let $x_{\rho}, \sum_{\varrho=1}^{m} q_{\varrho}(2 a-$ $\left.x_{\varrho}\right)$ and $\sum_{\rho=1}^{n} p_{\rho} \in \mathbb{I}_{2}$. Then

$$
\begin{aligned}
\tilde{J}(f(\cdot))= & \sum_{\varrho=1}^{m} q_{\varrho} f\left(2 a-x_{\varrho}\right)-f\left(\sum_{\varrho=1}^{m} q_{\varrho}\left(2 a-x_{\varrho}\right)\right)-\sum_{\rho=1}^{n} p_{\rho} f\left(x_{\rho}\right) \\
& +f\left(\sum_{\rho=1}^{n} p_{\rho} x_{\rho}\right) .
\end{aligned}
$$

Theorem 12 Assume $\mathcal{H}$ with $f \in C^{2 p+1}[0,2 a]$, and let $\Theta_{p}(t)$ be the same as defined in Lemma 1.1. Then, for $0 \leq \zeta_{1}<\zeta_{2} \leq 2 a$, we have

$$
\begin{aligned}
\tilde{J}(f(\cdot))= & \sum_{l=0}^{p-2}\left(\zeta_{2}-\zeta_{1}\right)^{2 l}\left[f^{(2 l+3)}\left(\zeta_{1}\right) \int_{\zeta_{1}}^{\zeta_{2}} \tilde{J}\left(G_{k}(\cdot, s)\right) \Theta_{l}\left(\frac{\zeta_{2}-s}{\zeta_{2}-\zeta_{1}}\right) d s\right. \\
& \left.+f^{(2 l+3)}\left(\zeta_{2}\right) \int_{\zeta_{1}}^{\zeta_{2}} \tilde{J}\left(G_{k}(\cdot, s)\right) \Theta_{l}\left(\frac{s-\zeta_{1}}{\zeta_{2}-\zeta_{1}}\right) d s\right]
\end{aligned}
$$




$$
\begin{aligned}
& +\left(\zeta_{2}-\zeta_{1}\right)^{2 p-3} \int_{\zeta_{1}}^{\zeta_{2}} f^{(2 p+1)}(v) \\
& \times\left(\int_{\zeta_{1}}^{\zeta_{2}} \breve{J}\left(G_{k}(\cdot, s)\right) G_{p}\left(\frac{s-\zeta_{1}}{\zeta_{2}-\zeta_{1}}, \frac{v-\zeta_{1}}{\zeta_{2}-\zeta_{1}}\right) d s\right) d v
\end{aligned}
$$

where

$$
\begin{aligned}
\tilde{J}\left(G_{k}(\cdot, s)\right)= & \sum_{\varrho=1}^{m} q_{\varrho} G_{k}\left(2 a-x_{\varrho}, s\right)-G_{k}\left(\sum_{\varrho=1}^{m} q_{\varrho}\left(2 a-x_{\varrho}, s\right)\right)-\sum_{\rho=1}^{n} p_{\rho} G_{k}\left(x_{\rho}, s\right) \\
& +G_{k}\left(\sum_{\rho=1}^{n} p_{\rho} x_{\rho}, s\right)
\end{aligned}
$$

and $G_{k}(\cdot, s)(k=1,2)$ are defined in (15) and (17) respectively.

Proof Replace $\mathbb{I}_{1}$ with $\mathbb{I}_{2}$ and $y_{\varrho}$ with $2 a-x_{\varrho}$ in Theorem 6, we get the required result.

In the following theorem we obtain generalizations of Levinson's inequality (for real weights) for $(2 p+1)$-convex functions.

Theorem 13 Assume that all the conditions of Theorem 12 hold, and let $f$ be a $(2 p+1)$ convex function. Then, for $k=1,2$ and $0 \leq \zeta_{1}<\zeta_{2} \leq 2 a$, we have the following inequalities:

If

$$
\int_{\zeta_{1}}^{\zeta_{2}} \tilde{J}\left(G_{k}(\cdot, s)\right) G_{p}\left(\frac{s-\zeta_{1}}{\zeta_{2}-\zeta_{1}}, \frac{v-\zeta_{1}}{\zeta_{2}-\zeta_{1}}\right) d s \geq 0
$$

then

$$
\begin{aligned}
\tilde{J}(f(\cdot)) \geq & \sum_{l=0}^{p-2}\left(\zeta_{2}-\zeta_{1}\right)^{2 l}\left[f^{(2 l+3)}\left(\zeta_{1}\right) \int_{\zeta_{1}}^{\zeta_{2}} \tilde{J}\left(G_{k}(\cdot, s)\right) \Theta_{l}\left(\frac{\zeta_{2}-s}{\zeta_{2}-\zeta_{1}}\right) d s\right. \\
& \left.+f^{(2 l+3)}\left(\zeta_{2}\right) \int_{\zeta_{1}}^{\zeta_{2}} \tilde{J}\left(G_{k}(\cdot, s)\right) \Theta_{l}\left(\frac{s-\zeta_{1}}{\zeta_{2}-\zeta_{1}}\right) d s\right] .
\end{aligned}
$$

Proof Similar to Theorem 7.

Remark 3 In Theorem 13, inequality in (38) holds in reverse direction if the inequality in (37) is reversed.

If we put $m=n, p_{\rho}=q_{\varrho}$ and use positive weights in (34), then $\tilde{J}(\cdot)$ converts to the functional $J_{1}(\cdot)$ defined in (2), also in this case (35), (36), (37), and (38) become, for 
$0 \leq \zeta_{1}<\zeta_{2} \leq 2 a$

$$
\begin{aligned}
& J_{1}(f(\cdot))= \sum_{l=0}^{p-2}\left(\zeta_{2}-\zeta_{1}\right)^{2 l}\left[f^{(2 l+3)}\left(\zeta_{1}\right) \int_{\zeta_{1}}^{\zeta_{2}} J_{1}\left(G_{k}(\cdot, s)\right) \Theta_{l}\left(\frac{\zeta_{2}-s}{\zeta_{2}-\zeta_{1}}\right) d s\right. \\
&\left.+f^{(2 l+3)}\left(\zeta_{2}\right) \int_{\zeta_{1}}^{\zeta_{2}} J_{1}\left(G_{k}(\cdot, s)\right) \Theta_{l}\left(\frac{s-\zeta_{1}}{\zeta_{2}-\zeta_{1}}\right) d s\right] \\
&+\left(\zeta_{2}-\zeta_{1}\right)^{2 p-3} \int_{\zeta_{1}}^{\zeta_{2}} f^{(2 p+1)}(v) \\
& \times\left(\int_{\zeta_{1}}^{\zeta_{2}} J_{1}\left(G_{k}(\cdot, s)\right) G_{p}\left(\frac{s-\zeta_{1}}{\zeta_{2}-\zeta_{1}}, \frac{v-\zeta_{1}}{\zeta_{2}-\zeta_{1}}\right) d s\right) d v, \\
& J_{1}\left(G_{k}(\cdot, s)\right)= \sum_{\rho=1}^{n} p_{\rho} G_{k}\left(2 a-x_{\varrho}, s\right)-G_{k}\left(\sum_{\rho=1}^{n} p_{\rho}\left(2 a-x_{\varrho}, s\right)\right)-\sum_{\rho=1}^{n} p_{\rho} G_{k}\left(x_{\rho}, s\right) \\
&+G_{k}\left(\sum_{\rho=1}^{n} p_{\rho} x_{\rho}, s\right), \\
& \int_{\zeta_{1}}^{\zeta_{2}} J_{1}\left(G_{k}(\cdot, s)\right) G_{p}\left(\frac{s-\zeta_{1}}{\zeta_{2}-\zeta_{1}}, \frac{\nu-\zeta_{1}}{\zeta_{2}-\zeta_{1}}\right) d s \geq 0,
\end{aligned}
$$

and

$$
\begin{aligned}
J_{1}(f(\cdot)) \geq & \sum_{l=0}^{p-2}\left(\zeta_{2}-\zeta_{1}\right)^{2 l}\left[f^{(2 l+3)}\left(\zeta_{1}\right) \int_{\zeta_{1}}^{\zeta_{2}} J_{1}\left(G_{k}(\cdot, s)\right) \Theta_{l}\left(\frac{\zeta_{2}-s}{\zeta_{2}-\zeta_{1}}\right) d s\right. \\
& \left.+f^{(2 l+3)}\left(\zeta_{2}\right) \int_{\zeta_{1}}^{\zeta_{2}} J_{1}\left(G_{k}(\cdot, s)\right) \Theta_{l}\left(\frac{s-\zeta_{1}}{\zeta_{2}-\zeta_{1}}\right) d s\right] .
\end{aligned}
$$

Theorem 14 Let $f: \mathbb{I}_{2}=[0,2 a] \rightarrow \mathbb{R}$ be a $(2 p+1)$-convex function. Also let $\left(p_{1}, \ldots, p_{n}\right)$ be positive real numbers such that $\sum_{\rho=1}^{n} p_{\rho}=1$. Then, for the functional $J_{1}(\cdot)$ defined in (3), and using $\Theta_{p}(t)$ defined in Lemma 1.1, we have the following:

(i) For $k=1,2$, inequality (42) holds provided that $p$ is odd.

(ii) For fixed $k=1,2$, let inequality (42) be satisfied and

$$
\sum_{l=0}^{p-2}\left(\zeta_{2}-\zeta_{1}\right)^{2 l}\left[f^{(2 l+3)}\left(\zeta_{1}\right) \Theta_{l}\left(\frac{\zeta_{2}-s}{\zeta_{2}-\zeta_{1}}\right)+f^{(2 l+3)}\left(\zeta_{2}\right) \Theta_{l}\left(\frac{s-\zeta_{1}}{\zeta_{2}-\zeta_{1}}\right)\right] \geq 0
$$

Then

$$
J_{1}(f(\cdot)) \geq 0
$$

Proof By using Theorem 13 and Remark 1.

Remark 4 Cebyšev, Grüss, and Ostrowski type new bounds related to the obtained generalizations can also be discussed. Moreover, we can also give related mean value theorems by using non-negative functionals (20) and (35) to construct the new families of $n$-exponentially convex functions and Cauchy means related to these functionals as given in Sect. 4 of [23]. 


\section{Estimation of $f$-divergence and Shannon entropy}

In this section we obtain applications of information theory. We apply Theorem 7 for $(2 p+1)$-convex functions to $\hat{\mathbb{I}}_{f}(\tilde{\mathbf{r}}, \tilde{\mathbf{k}})$.

Theorem 15 Let $\tilde{\mathbf{r}}=\left(r_{1}, \ldots, r_{n}\right) \in \mathbb{R}^{n}, \tilde{\mathbf{w}}=\left(w_{1}, \ldots, w_{m}\right) \in \mathbb{R}^{m}, \tilde{\mathbf{k}}=\left(k_{1}, \ldots, k_{n}\right) \in(0, \infty)^{n}$, and $\tilde{\mathbf{t}}=\left(t_{1}, \ldots, t_{m}\right) \in(0, \infty)^{m}$ be such that

$$
\frac{r_{\rho}}{k_{\rho}} \in \mathbb{I}, \quad \rho=1, \ldots, n,
$$

and

$$
\frac{w_{\varrho}}{t_{\varrho}} \in \mathbb{I}, \quad \varrho=1, \ldots, m
$$

Also let $f \in C^{2 p+1}\left[\zeta_{1}, \zeta_{2}\right]$ be such that $f$ is $(2 p+1)$-convex (for odd $\left.p\right)$ function, then

$$
\begin{aligned}
J_{c i s}(f(\cdot)) \geq & \sum_{l=0}^{p-2}\left(\zeta_{2}-\zeta_{1}\right)^{2 l}\left[f^{(2 l+3)}\left(\zeta_{1}\right) \int_{\zeta_{1}}^{\zeta_{2}} J\left(G_{k}(\cdot, s)\right) \Theta_{l}\left(\frac{\zeta_{2}-s}{\zeta_{2}-\zeta_{1}}\right) d s\right. \\
& \left.+f^{(2 l+3)}\left(\zeta_{2}\right) \int_{\zeta_{1}}^{\zeta_{2}} J\left(G_{k}(\cdot, s)\right) \Theta_{l}\left(\frac{s-\zeta_{1}}{\zeta_{2}-\zeta_{1}}\right) d s\right]
\end{aligned}
$$

for $k=1,2$

$$
\begin{aligned}
J\left(G_{k}(\cdot, s)\right)= & \sum_{\varrho=1}^{m} \frac{t_{\varrho}}{\sum_{\varrho=1}^{m} t_{\varrho}} G_{k}\left(\frac{w_{\varrho}}{t_{\varrho}}, s\right)-G_{k}\left(\sum_{\varrho=1}^{m} \frac{w_{\varrho}}{\sum_{\varrho=1}^{m} t_{\varrho}}, s\right)-\sum_{\rho=1}^{n} \frac{k_{\rho}}{\sum_{\rho=1}^{n} k_{\rho}} G_{k}\left(\frac{r_{\rho}}{k_{\rho}}, s\right) \\
& +G_{k}\left(\sum_{\rho=1}^{n} \frac{r_{\rho}}{\sum_{\rho=1}^{n} k_{\rho}}, s\right) .
\end{aligned}
$$

Proof It is clear that Green's functions $G_{k}(\cdot, s)$ defined in (15) and (17) are 3-convex, therefore $J\left(G_{k}(\cdot, s)\right) \geq 0$ for fixed $k=1,2$. Also $G_{p}\left(\frac{s-\zeta_{1}}{\zeta_{2}-\zeta_{1}}, \frac{v-\zeta_{1}}{\zeta_{2}-\zeta_{1}}\right) \geq 0$ for odd $p$, therefore (26) holds. Hence, using $p_{\rho}=\frac{k_{\rho}}{\sum_{\rho=1}^{n} k_{\rho}}, x_{\rho}=\frac{r_{\rho}}{k_{\rho}}, q_{\varrho}=\frac{t_{\varrho}}{\sum_{\varrho=1}^{m} t_{\varrho}}, y_{\varrho}=\frac{w_{\varrho}}{t_{\varrho}}$ in Theorem 7, (27) becomes (45), where $\hat{\mathbb{I}}_{f}(\tilde{\mathbf{r}}, \tilde{\mathbf{k}})$ is defined in (1) and

$$
\hat{\mathbb{I}}_{f}(\tilde{\mathbf{w}}, \tilde{\mathbf{t}}):=\sum_{\varrho=1}^{m} t_{\varrho} f\left(\frac{w_{\varrho}}{t_{\varrho}}\right) .
$$

\subsection{Shannon entropy}

Definition 3 (see [10]) The $\mathcal{S}$ hannon entropy of positive probability distribution $\tilde{\mathbf{k}}=$ $\left(k_{1}, \ldots, k_{n}\right)$ is defined by

$$
\mathcal{S}:=-\sum_{\rho=1}^{n} k_{\rho} \log \left(k_{\rho}\right) .
$$

Corollary 2 Let $\tilde{\mathbf{k}}=\left(k_{1}, \ldots, k_{n}\right)$ and $\tilde{\mathbf{t}}=\left(t_{1}, \ldots, t_{m}\right)$ be positive probability distributions. Also let $\tilde{\mathbf{r}}=\left(r_{1}, \ldots, r_{n}\right) \in(0, \infty)^{n}$ and $\tilde{\mathbf{w}}=\left(w_{1}, \ldots, w_{m}\right) \in(0, \infty)^{m}$. 
If base of $\log$ is greater than 1 and $p=\operatorname{odd}(p>2)$, then

$$
\begin{aligned}
J_{s}(\cdot) \geq & \sum_{l=0}^{p-2}\left(\zeta_{2}-\zeta_{1}\right)^{2 l}\left[\frac{(-1)^{2 l+2}(2 l+2) !}{\left(\zeta_{1}\right)^{2 l+3}} \int_{\zeta_{1}}^{\zeta_{2}} J\left(G_{k}(\cdot, s)\right) \Theta_{l}\left(\frac{\zeta_{2}-s}{\zeta_{2}-\zeta_{1}}\right) d s\right. \\
& \left.+\frac{(-1)^{2 l+2}(2 l+2) !}{\left(\zeta_{1}\right)^{2 l+3}} \int_{\zeta_{1}}^{\zeta_{2}} J\left(G_{k}(\cdot, s)\right) \Theta_{l}\left(\frac{s-\zeta_{1}}{\zeta_{2}-\zeta_{1}}\right) d s\right]
\end{aligned}
$$

where

$$
\begin{aligned}
J_{s}(\cdot)= & \sum_{\varrho=1}^{m} t_{\varrho} \log \left(w_{\varrho}\right)+\tilde{\mathcal{S}}-\log \left(\sum_{\varrho=1}^{m} w_{\varrho}\right)-\sum_{\rho=1}^{n} k_{\rho} \log \left(r_{\rho}\right)+\mathcal{S} \\
& +\log \left(\sum_{\rho=1}^{n} r_{\rho}\right)
\end{aligned}
$$

and for fixed $k=1,2, J\left(G_{k}(\cdot, s)\right)$ is the same as defined in (46).

Proof The function $f: x \rightarrow \log (x)$ is $(2 p+1)$-convex for odd $p(p>1)$ and base of $\log$ is greater than 1 . Therefore we use $f=\log (x)$ in (45) to get (49), where $\mathcal{S}$ is defined in (48) and

$$
\tilde{\mathcal{S}}=-\sum_{\varrho=1}^{m} t_{\varrho} \log \left(t_{\varrho}\right)
$$

\section{Acknowledgements}

The authors wish to thank the anonymous referees for their very careful reading of the manuscript and fruitful comments and suggestions. The research of the 4th author is supported by the Ministry of Education and Science of the Russian Federation (the Agreement number No. 02.a03.21.0008).

Funding

There is no funding for this work.

\section{Competing interests}

The authors declare that there is no conflict of interests regarding the publication of this paper.

\section{Authors' contributions}

All authors contributed equally. All authors jointly worked on the results and they read and approved the final manuscript.

\section{Author details}

${ }^{1}$ Department of Mathematics, University of Sargodha, Sargodha, Pakistan. ${ }^{2}$ Department of Mathematics, University of

Central Punjab, Lahore, Pakistan. ${ }^{3}$ Catholic University of Croatia, Zagreb, Croatia. ${ }^{4}$ RUDN University, Moscow, Russia.

\section{Publisher's Note}

Springer Nature remains neutral with regard to jurisdictional claims in published maps and institutional affiliations.

Received: 30 October 2019 Accepted: 8 January 2020 Published online: 14 January 2020

\section{References}

1. Liese, F., Vajda, I.: Convex Statistical Distances. Teubner-Texte Zur Mathematik, vol. 95. Teubner, Leipzig (1987)

2. Vajda, I.: Theory of Statistical Inference and Information. Kluwer Academic, Dordrecht (1989)

3. Adeel, M., Khan, K.A., Pečarić, Đ., Pečarić, J.: Generalization of the Levinson inequality with applications to information theory. J. Inequal. Appl. 2019, 230 (2019)

4. Khan, K.A., Niaz, T., Pečarić, Đ., Pečarić, J.: Refinement of Jensen's inequality and estimation of $f$-and Rényi divergence via Montgomery identity. J. Inequal. Appl. 2018, 318 (2018)

5. Adeel, M., Khan, K.A., Pečarić, Đ., Pečarić, J.: Levinson type inequalities for higher order convex functions via Abel-Gontscharoff interpolation. Adv. Differ. Equ. 2019,430 (2019)

6. Gibbs, A.L.: On choosing and bounding probability metrics. Int. Stat. Rev. 70, 419-435 (2002) 
7. Sason, I., Verdú, S.: f-Divergence inequalities. IEEE Trans. Inf. Theory 62, 5973-6006 (2016)

8. Csiszár, I.: Information measures: a critical survey. In: Tans. 7th Prague Conf. on Info. Th., Statist. Decis. Funct., Random Process and 8th European Meeting of Statist., vol. B, pp. 73-86. Academia, Prague (1978)

9. Csiszár, I.: Information-type measures of difference of probability distributions and indirect observations. Studia Sci. Math. Hung. 2, 299-318 (1967)

10. Horváth, L.,Pečarić, Đ.,Pečarić, J.: Estimations of $f$ - and Rényi divergences by using a cyclic refinement of the Jensen's inequality. Bull. Malays. Math. Sci. Soc. 42, 933-946 (2019)

11. Pečarić, J., Proschan, F., Tong, Y.L.: Convex Functions, Partial Orderings and Statistical Applications. Academic Press, New York (1992)

12. Levinson, N.: Generalization of an inequality of Kay Fan. J. Math. Anal. Appl. 6, 133-134 (1969)

13. Mitrinović, D.S., Pečarić, J., Fink, A.M.: Classical and New Inequalities in Analysis, vol. 61. Kluwer Academic, Dordrecht (1991)

14. Popoviciu, T.: Sur une inegalite de N. Levinson. Mathematica 6, 301-306 (1969)

15. Bullen, P.S.: An inequality of N. Levinson. Univ. Beogr. Publ. Elektroteh. Fak. Ser. Mat. Fiz. 412-460, 109-112 (1973)

16. Pečarić, J.: On an inequality on N. Levinson. Univ. Beogr. Publ. Elektroteh. Fak. Ser. Mat. Fiz. 678-715, 71-74 (1980)

17. Mercer, A.M.: A variant of Jensen's inequality. J. Inequal. Pure Appl. Math. 4(4), Article 73 (2003)

18. Widder, D.V.: Completely convex function and Lidstone series. Trans. Am. Math. Soc. 51, 387-398 (1942)

19. Agarwal, R.P., Wong, P.J.Y.: Error Inequalities in Polynomial Interpolation and Their Applications. Springer, Berlin (1992)

20. Aras Gazić, G., Culjak, V., Pečarić, J., Vukelić, A.: Generalization of Jensen's inequality by Lidstone's polynomial and related results. Math. Inequal. Appl. 164, 1243-1267 (2013)

21. Pečarić, J., Praljak, M., Witkowski, A.: Generalizes Levinson's inequality and exponential convexity. Opusc. Math. 35 397-410 (2015)

22. Pečarić, J., Praljak, M., Witkowski, A.: Linear operators inequality for $n$-convex functions at a point. Math. Inequal. Appl. 18, 1201-1217 (2015)

23. Butt, S.I., Khan, K.A., Pečarić, J.: Generalization of Popoviciu inequality for higher order convex function via Taylor's polynomial. Acta Univ. Apulensis, Mat.-Inform. 42, 181-200 (2015)

\section{Submit your manuscript to a SpringerOpen ${ }^{\circ}$ journal and benefit from:}

- Convenient online submission

- Rigorous peer review

Open access: articles freely available online

- High visibility within the field

- Retaining the copyright to your article

Submit your next manuscript at $>$ springeropen.com 